\title{
Frequency support by scheduling of variable-speed wind turbines
}

\author{
A. De Paola* D. Angeli ${ }^{* *}$ G. Strbac ${ }^{* * *}$ \\ * Electrical and Electronic Engineering Department, Imperial College \\ London, UK, (e-mail: antonio.de-paola09@imperial.ac.uk) \\ ** Electrical and Electronic Engineering Department, Imperial College \\ London, UK, (e-mail: d.angeli@imperial.ac.uk) \\ *** Electrical and Electronic Engineering Department, Imperial College \\ London, UK, (e-mail: g.strbac@imperial.ac.uk)
}

\begin{abstract}
This paper characterizes optimal control policies for wind farms operated as frequency response services in case of a fault of conventional generators. The frequency support is provided through temporary over-production: when frequency drops, the turbines move from the steady-state operating point and extra power is produced by slowing down the turbines and releasing part of their kinetic energy. The control task is formulated and solved as an optimal containment problem: the time during which an extra quantity of power can be produced, within the set speed constraints for each turbine, is maximized. The solutions are calculated and compared for different assumptions on the electric torque of the turbines.
\end{abstract}

\section{INTRODUCTION}

In a power system the network frequency is an indicator of the balance which is kept at all times between supply and demand. Imbalances may be introduced by trips of large generating plants which cause a sudden drop in supply. The corresponding drop in frequency is initially mitigated by the inertial response, as described in Kundur (1994): synchronous generators slow down and release part of their kinetic energy. The growing environmental concerns and advancements in technology are causing an increasing penetration of asynchronous wind generation in power systems. One significant effect of this phenomenon will be the reduction of the system inertia: Lalor et al. (2005) shows that steeper frequency falls will occur in case of a fault in the network. Hence it will be of major importance that wind turbines provide frequency response services in the near future. Many approaches for frequency support with wind turbines have been proposed, such as the release of kinetic energy when frequency drops are detected in Ullah et al. (2008) or the introduction of a deloaded maximum power extraction curve in de Almeyda and Lopes (2007). What is proposed in this paper is a scheduling of the wind generators: the electric torque applied to each turbine when a fault occurs is chosen in order for the whole population to generate an extra quantity of power. The scheduling design has been approached as an optimal containment problem: the additional power is produced for as long as possible and, at the same time, the rotor speed of the turbines must always be within the operational boundaries. Similar problems, arising for example in the control of surge tanks, have been tackled using different approaches, such as differential games in Falugi et al. (2012) and Boué and Dupuis (2001) or predictive control in Ogawa et al. (2002). This paper is structured as follows: in

\footnotetext{
* The work is partially supported by FP7 grant 283145 "ClusterDesign".
}

Section 2 the single wind generator and its efficiency with respect to rotor speed are modelled. The first approach for frequency support, where each turbine can operate in two different modes, is presented in Section 3, while its results are shown through simulations in Section 4. The case where the electric torque can be arbitrarily chosen for all turbines is studied in Section 5 .

\section{MODELLING}

The single wind turbine has been modelled in its mechanical part as a rotating mass, describing its angular speed $\omega$ by the swing equation:

$$
\dot{\omega}=\frac{1}{J}\left(T_{m}-T_{e}\right)
$$

where $J$ is the total moment of inertia, $T_{m}$ is the mechanical torque extracted from the wind and $T_{e}$ is the electric torque used to generate power. If we consider the wind speed $v$ and we denote by $R$ the radius of the rotor and by $\mu$ the air density, the power of the wind $P_{w}$ and the corresponding mechanical torque are:

$$
P_{w}=\frac{\mu \pi R^{2} v^{3}}{2} T_{m}=\frac{P_{w} C_{P}(\lambda, \theta)}{\omega}
$$

where the power coefficient $C_{P}$ is dependent on the tipspeed ratio $\lambda=\frac{\omega R}{v}$ and the pitch angle $\theta$. The expression of $C_{P}$ adopted in our simulations is the one used by Slootweg et al. (2001):

$$
\begin{gathered}
C_{P}(\lambda, \theta)=0.22\left(\frac{116}{\lambda_{i}}-0.4 \theta-5\right) e^{-\frac{12.5}{\lambda_{i}}} \\
\frac{1}{\lambda_{i}}=\frac{1}{\lambda+0.08 \theta}-\frac{0.035}{\theta^{3}+1}
\end{gathered}
$$

We initially assume, for the sake of simplicity, that the wind speed $v$ is constant. For low wind conditions pitch angle actions are not applied and the angle $\theta$ is constant and equal to zero. Under such assumptions, the $C_{P}$ coefficient is only dependent from the rotor speed $\omega$. 
Remark 1. It is reasonable to assume, by considering the expressions of $C_{P}$ used in the literature, that the power coefficient has a maximum point for some rotor speed $\omega=\omega_{0}$ and an inflection point for $\omega_{F}<\omega_{0}$.

Furthermore, the electrical dynamics of the turbine have been neglected, assuming that the electric torque is the input of the considered system. We propose alternative solutions to the scheduling problem under different assumptions:

(1) Each turbine can only operate in two modes, one corresponding to the steady state at maximum power extraction and one corresponding to a slower suboptimal equilibrium. By switching to the second mode the wind turbine reduces its kinetic energy and is able to temporarily generate more power. This policy can be desirable if one wants to slow down only a fraction of the wind farm while waiting for secondary response services to take over. Note that in this case, in order to coordinate the switching between the two modes of each generator, centralized management of the turbines is required.

(2) Each turbine can be arbitrarily slowed down or accelerated. We will show that under this assumption the optimal policy can be implemented in a decentralized way.

The first case is analysed in Section 3 and the second approach is detailed in Section 5 .

\section{TWO-MODES SCHEDULING}

\subsection{General framework}

The two different modes in which each turbine can operate (respectively denoted by plus and minus subscripts), correspond to different choices of $T_{e}$. In one case it is desirable to operate at the rotor speed $\omega_{0}$ (and at the resulting tipspeed ratio $\lambda_{0}=\omega_{0} R / v$ ) that maximizes the $C_{P}$ coefficient and guarantees optimal efficiency. This is usually done by choosing $T_{e}$ as the function $T_{+}$specified in Burton et al. (2001):

$$
T_{+}(\omega)=\frac{\mu \pi R^{5} C_{P}\left(\omega_{0}\right)}{2 \lambda_{0}^{3}} \omega^{2}=K_{T} \omega^{2}
$$

Denoting by $\omega_{+}$the rotor speed in the first operative mode and replacing (2) and (4) in the swing equation (1), we obtain:

$$
\dot{\omega}_{+}=\frac{P_{w}}{J} \frac{\omega_{0}^{3} C_{P}\left(\omega_{+}\right)-\omega_{+}^{3} C_{P}\left(\omega_{0}\right)}{\omega_{0}^{3} \omega_{+}}
$$

It is straightforward to notice that $\omega_{0}$ is an equilibrium point for (5) and it can be easily proved, by linearization of the system, that such equilibrium is locally asymptotically stable. The wind turbines are able to provide frequency response by switching to the second operative mode, where an additional torque $\tau$ is applied, slowing down the turbine and returning part of its kinetic energy to the network:

$$
\dot{\omega}_{-}=\frac{P_{w}}{J} \frac{\omega_{0}^{3} C_{P}\left(\omega_{-}\right)-\omega_{-}^{3} C_{P}\left(\omega_{0}\right)}{\omega_{0}^{3} \omega_{-}}-\frac{\tau}{J}
$$

Particular care must be taken in the choice of $\tau$ : the operative constraints on the rotor speed $\omega$ can be satisfied by choosing the additional torque $\tau$ in such a way that the suboptimal equilibrium speed for (6) is acceptable. High values of $\tau$ must also be avoided, in order to limit the mechanical stress on the switching turbines.

Given their particular structure and the complicated expression of $C_{P}$, it has not been possible to solve the equations (5) and (6) analytically, however, their solutions are straightforward to obtain numerically. If we denote by $\phi_{+}\left(\omega_{I N}, t\right)$ and $\phi_{-}\left(\omega_{I N}, t\right)$ the solutions at time $t$ with initial condition $\omega(0)=\omega_{I N}$, the equivalent power generated by the turbine in the two modes is:

$$
\begin{gathered}
P_{+}\left(\omega_{I N}, t\right)=K_{T} \phi_{+}^{3}\left(\omega_{I N}, t\right) \\
P_{-}\left(\omega_{I N}, t\right)=K_{T} \phi_{-}^{3}\left(\omega_{I N}, t\right)+\tau \phi_{-}\left(\omega_{I N}, t\right)
\end{gathered}
$$

We consider a population of wind turbines where each element can switch between the two operative modes and all turbines are initially operating in the first mode, at the optimal rotor speed $\omega_{0}$. The control input $\rho(t)$ will represent the rate at which turbines switch to the second operative mode at time $t$. We will assume that $\int \rho(t) d t \in[0,1]$ which constitutes a reasonable approximation if the number of considered turbines is very high. The elements that switch to the second mode at $t$ will remain in such mode for $T(t)$ seconds, where $T$ is a second control input. Notice that turbines switching at the same time are constrained to commute back to the original mode simultaneously. This hypothesis makes the analysis of the system easier and does not limit its performance, as we will show later on.

We now want to understand what is the choice of $\rho(t)$ and $T(t)$ that guarantees the best frequency response. We will do so by considering the following scenario: a fault in the network is detected at $t=0$ and a fraction $\rho_{0}$ of turbines switches to the second mode, instantly achieving the corresponding power variation $\Delta P=\tau \omega_{0} \rho_{0}$. This case can be modelled by considering $\rho(t)$ presenting a Dirac pulse of amplitude $\rho_{0}$ at $t=0$. Our optimality criterion will be the maximization of the time interval $T_{E N D}$ during which the power generated by the wind farm can be kept greater or equal than $P_{\text {des }}=P_{0}+\Delta P$. We study a similar problem which is easier to solve and is obtained imposing $P(t)=P_{\text {des }}$ or, equivalently, $\dot{P}(t)=0$ in the interval $\left[0, T_{E N D}\right]$. Denoting by $\mathbb{D}$ the set of integrable distributions with non negative values, the optimal problem becomes:

$$
\begin{array}{ll} 
& \max T_{E N D} \\
\text { s.t. } & \int_{0^{+}, T(\cdot), T_{E N D}}^{T_{E N D}} \rho(t) d t=1-\rho_{0} \\
& \dot{P}(t)=0 \forall t \in\left[0, T_{E N D}\right] \\
& \rho(\cdot) \in \mathbb{D}
\end{array}
$$

In order to calculate the optimal solution $\rho^{*}(t)$ and $T^{*}(t)$ a slightly different case, obtained by removing the integral constraint on $\rho(t)$, is preliminarily studied:

$$
\begin{array}{ll} 
& \min \int^{T_{E N D}(\cdot), T(\cdot)} \int_{0^{+}}^{T_{E}} \rho(t) d t \\
\text { s.t. } & \dot{P}(t)=0 \forall t \in\left[0, T_{E N D}\right] \\
& \rho(\cdot) \in \mathbb{D}
\end{array}
$$

Some additional notation and properties which are required for the resolution of these two problems are detailed next while the main results are provided in section 3.3. 


\subsection{Preliminary operations}

The power generated by a single turbine at time $t$ if this has switched to the second mode for $t=0$ and switched back to the first mode at $t=T$ is denoted by $P_{G}(t, T)$ :

$$
P_{G}(t, T)=\left\{\begin{array}{cc}
P_{-}\left(\omega_{0}, t\right) & \text { if } t<T \\
P_{+}\left(\phi_{-}\left(\omega_{0}, T\right), t-T\right) & \text { if } t \geq T
\end{array}\right.
$$

Thanks to the previous expression, it is straightforward to derive the rate of switching $\eta(t, T)$ that compensates the power variation introduced at time $t$ by a unitary pulse of turbines switching at 0 and undergoing a switch back to the initial mode after $T$ seconds:

$$
\eta(t, T)=-\frac{1}{\tau \omega_{0}} \frac{\partial P_{G}(t, T)}{\partial t}
$$

Notice that the time derivative of $P_{G}$ is continuous everywhere, except for a Dirac delta of amplitude $-\tau \phi_{-}\left(\omega_{0}, T\right)$ at $t=T$. It is important to point out that in this scenario $\rho(t)$ is generally defined by the constraint on the power derivative and the choice of $T(\cdot)$ :

$$
\rho(t)=\int_{0}^{t_{-}} \eta(t-\tau, T(\tau)) \rho(\tau) d \tau
$$

This means that solution of problem (9) amounts to optimally select $T(\cdot)$. It is possible to extend our notation and, for a given $T(\cdot)$, denote by $\bar{\rho}(t, s)$ the rate of switching after $t$ seconds if a unit impulse of turbines undergoes switching at time $s$ and the power is kept constant thereafter. By noticing that, for a given time instant, $\bar{\rho}(t, s)$ must compensate the power losses introduced by all turbines that have switched in $[s, s+t)$, we obtain the following definition:

$$
\begin{aligned}
\bar{\rho}(t, s) & =\int_{0}^{t_{-}} \eta(t-\tau, T(s+\tau)) \bar{\rho}(\tau, s) d \tau \\
& =\eta(t, T(s))+\int_{0_{+}}^{t_{-}} \eta(t-\tau, T(s+\tau)) \bar{\rho}(\tau, s) d \tau
\end{aligned}
$$

An alternative expression for $\bar{\rho}(t, s)$ can be obtained by considering that, at each time instant $t$, two different components must be taken into account:

(1) The turbines that switch in order to compensate the initial power variation: $\eta(t, T(s))$

(2) The turbines that compensate the cascaded losses introduced at each time instant $\tau<t$ by the first component above: $\eta(\tau, T(s)) \bar{\rho}(t-\tau, s+\tau)$

Therefore we can write:

$$
\begin{aligned}
\bar{\rho}(t, s) & =\eta(t, T(s))+\int_{0_{+}}^{t_{-}} \eta(\tau, T(s)) \bar{\rho}(t-\tau, s+\tau) d \tau \\
& =\int_{0_{+}}^{t} \eta(\tau, T(s)) \bar{\rho}(t-\tau, s+\tau) d \tau
\end{aligned}
$$

A similar dual definition can be introduced for the integral $\bar{\rho}_{I}(t, s)$, defined as the cumulative fraction of turbines that switch in the interval $(s, s+t]$, for a given $T(\cdot)$, if a unit impulse of turbines undergoes switching at $s$ and the total generated power is to be kept constant:

$$
\bar{\rho}_{I}(t, s)=\int_{0^{+}}^{t} \bar{\rho}(\tau, s) d \tau
$$

Lemma 1. The integral $\bar{\rho}_{I}$ can be alternatively defined as:

$$
\bar{\rho}_{I}(t, s)=\int_{0^{+}}^{t} \eta(\tau, T(s))\left[1+\bar{\rho}_{I}(t-\tau, s+\tau)\right] d s
$$

To show the equivalence of the two definitions of $\bar{\rho}_{I}$, we initially replace (14) in (15):

$$
\begin{aligned}
\bar{\rho}_{I}(t) & =\int_{0^{+}}^{t} \eta(\tau, T(s)) d \tau \\
& +\int_{0^{+}}^{t} \int_{0^{+}}^{\tau_{-}} \eta(x, T(s)) \bar{\rho}(\tau-x, s+x) d x d \tau
\end{aligned}
$$

By switching the order of integration in the second term and introducing the change of variable $\tilde{\tau}=\tau-x$, we obtain:

$$
\begin{aligned}
\bar{\rho}_{I}(t, s) & =\int_{0^{+}}^{t} \eta(\tau, T(s)) d \tau \\
& +\int_{0^{+}}^{t} \int_{0^{+}}^{t-x} \eta(x, T(s)) \bar{\rho}(\tilde{\tau}, s+x) d \tilde{\tau} d x
\end{aligned}
$$

It is easy to realize that, moving $\eta(x, T(s))$ out of the second integral, the resulting expression is equal to (16).

\subsection{Optimal scheduling}

We can now state the main result for this section:

Theorem 1. The optimal switching profile $\tilde{T}(t)$ for the optimization problem (9) is given by:

$\tilde{T}(t)=\underset{T \in\left(0, T_{E N D}-t\right]}{\arg \min }\left[\int_{0^{+}}^{T_{E N D}-t} \eta(s, T)\left[1+\tilde{\rho}_{I}(t+s)\right] d s\right]$

where $\tilde{\rho}_{I}$ is defined by the following integral equation solved backwards in time (with $\left.\tilde{\rho}_{I}\left(T_{E N D}\right)=0\right)$ :

$$
\tilde{\rho}_{I}(t)=\int_{0^{+}}^{T_{E N D}-t} \eta(s, \tilde{T}(t))\left[1+\tilde{\rho}_{I}(t+s)\right] d s
$$

Proof. We initially notice that the objective function in (9) is equal to $\rho_{0} \bar{\rho}_{I}\left(T_{E N D}, 0\right)$, which in turn is a function of the switching time profile $T(\cdot)$. When this is chosen equal to $\tilde{T}(\cdot)$ it yields:

$$
\tilde{\rho}_{I}(t)=\bar{\rho}_{I}\left(T_{E N D}-t, t\right)
$$

It is now necessary to show that $\rho_{0} \tilde{\rho}_{I}(0)$ is the minimum for the considered optimization problem. In order to so, we introduce a different switching profile $\hat{T}(\cdot)$ which does not satisfy condition (19) on a set $\mathcal{T} \subseteq\left[0, T_{E N D}\right]$ of positive measure. The resulting cost function $\hat{\rho}_{I}$, with $\hat{\rho}_{I}\left(T_{E N D}\right)=0$, is given by:

$$
\hat{\rho}_{I}(t)=\int_{0^{+}}^{T_{E N D}-t} \eta(s, \hat{T}(t))\left[1+\hat{\rho}_{I}(t+s)\right] d s
$$

If we introduce $\rho_{D}^{A}(t)=\hat{\rho}_{I}(t)-\tilde{\rho}_{I}(t)$, subtracting (20) from (22) we obtain:

$$
\begin{aligned}
\rho_{D}^{A}(t) & =\int_{0^{+}}^{T_{E N D}-t}[\eta(s, \hat{T}(t))-\eta(s, \tilde{T}(t))]\left[1+\tilde{\rho}_{I}(t+s)\right] d s \\
& +\int_{0^{+}}^{T_{E N D}-t} \eta(s, \hat{T}(t)) \rho_{D}^{A}(t+s) d s \\
& =\rho_{D}^{0}(t)+\int_{0^{+}}^{T_{E N D}-t} \eta(s, \hat{T}(t)) \rho_{D}^{A}(t+s) d s
\end{aligned}
$$

Notice that, following the definition of $\tilde{T}$ in (19), we have $\rho_{D}^{0}(t)>0 \forall t \in \mathcal{T}$ and $\rho_{D}^{0}(t)=0$ elsewhere. An equivalent 
expression for the difference between the two cost functions is given by $\rho_{D}^{B}$, defined as follows:

$$
\rho_{D}^{B}(t)=\int_{t}^{T_{E N D}} \bar{\rho}_{D}(t, s) d s
$$

where $\bar{\rho}_{D}(t, s)$ represents the component of $\rho_{D}^{B}(t)$ resulting by different values of $\tilde{T}$ and $\hat{T}$ at time $s$ :

$$
\bar{\rho}_{D}(t, s)=\rho_{D}^{0}(t) \delta(t-s)+\int_{0^{+}}^{s-t} \eta(\tau, \hat{T}(t)) \bar{\rho}_{D}(t+\tau, s) d \tau
$$

For $s \notin \mathcal{T}$ and $t \leq s$ we have that $\bar{\rho}_{D}(t, s)=0$ is a solution of (25). To prove the equivalence of $\rho_{D}^{A}$ and $\rho_{D}^{B}$ we initially notice that they are both equal to 0 for $t=T_{E N D}$ and then we show that $\rho_{D}^{B}$ satisfies the integral definition in $(23)$ :

$$
\begin{aligned}
\rho_{D}^{B}(t) & =\rho_{D}^{0}(t)+\int_{t^{+}}^{T_{E N D}} \bar{\rho}_{D}(t, \tau) d \tau \\
& =\rho_{D}^{0}(t)+\int_{t^{+}}^{T_{E N D}} \int_{0^{+}}^{\tau-t} \eta(s, \hat{T}(t)) \bar{\rho}_{D}(t+s, \tau) d s d \tau \\
& =\rho_{D}^{0}(t)+\int_{0^{+}}^{T_{E N D}} \int_{t+s}^{T_{E N D}} \eta(s, \hat{T}(t)) \bar{\rho}_{D}(t+s, \tau) d \tau d s \\
& =\rho_{D}^{0}(t)+\int_{0^{+}}^{T_{E N D}-t} \eta(s, \hat{T}(t)) \rho_{D}^{B}(t+s) d s
\end{aligned}
$$

We now introduce $\overline{\bar{\rho}}(t, s)=\bar{\rho}(s-t, t)$ using (14):

$$
\overline{\bar{\rho}}(t, s)=\int_{0_{+}}^{s-t} \eta(\tau, T(t)) \overline{\bar{\rho}}(t+\tau, s) d s
$$

It is easy to notice that $\bar{\rho}_{D}$ and $\overline{\bar{\rho}}$ fulfill the same integral equation. This means that under the considered overproduction regime, since $\rho_{D}^{0}(s)>0 \forall s \in \mathcal{T}$, the corresponding $\bar{\rho}_{D}(\cdot, s)$ are always greater than 0 . Since $\bar{\rho}_{D}(\cdot, s)=0$ $\forall s \notin \mathcal{T}$, we can conclude that $\rho_{D}^{B}(0)>0$ and therefore $\rho_{0} \tilde{\rho}_{I}(0)$ is the minimum for the cost function in (9).

Remark 2. The hypothesis that the time $T(t)$ of permanence in mode 2 is equal for all turbines is not restrictive. Assume that, for $t=\bar{t}$, the turbines are divided in two groups of size $k_{1}$ and $k_{2}$ that switch back for $T_{1}(\bar{t})$ and $T_{2}(\bar{t})$, which may differ in general. The optimization problem becomes:

$$
\begin{aligned}
\min _{T_{1}, T_{2}} & {\left[\int_{0^{+}}^{T_{E N D}-\bar{t}} k_{1} \eta\left(s, T_{1}\right) \cdot\left[1+\tilde{\rho}_{I}(t+s)\right]\right.} \\
& \left.+k_{2} \eta\left(s, T_{2}\right) \cdot\left[1+\tilde{\rho}_{I}(t+s)\right] d s\right]
\end{aligned}
$$

The solution can be obtained by separately solving two optimization problems of the same form:

$$
\begin{aligned}
& \min _{T_{1}}\left[k_{1} \int_{0^{+}}^{T_{E N D}-\bar{t}} \eta\left(s, T_{1}\right) \cdot\left[1+\tilde{\rho}_{I}(t+s)\right]\right] \\
& \min _{T_{2}}\left[k_{2} \int_{0^{+}}^{T_{E N D}-\bar{t}} \eta\left(s, T_{2}\right) \cdot\left[1+\tilde{\rho}_{I}(t+s)\right]\right]
\end{aligned}
$$

Hence they will yield the same results and this shows that considering a unique switching policy does not degrade the optimal solution.

Remark 3. We denote by $\tilde{I}\left(T_{E N D}\right)$ and $\tilde{T}_{S}\left(T_{E N D}, \cdot\right)$ respectively the optimal value and optimal switching times for (9) as functions of the considered time interval $T_{E N D}$ If the minimization is performed for a certain $T_{E N D}=T_{1}$ by solving (19) and (20) backwards in time, the solutions for all $T_{2}<T_{1}$ are also obtained:

$$
\tilde{I}\left(T_{2}\right)=\rho_{0} \cdot \tilde{\rho}_{I}\left(T_{1}-T_{2}\right) \tilde{T}_{S}\left(T_{2}, t\right)=\tilde{T}_{S}\left(T_{1}, t+T_{1}-T_{2}\right)
$$

It is easy to notice, from (20) and (30), that $\tilde{I}\left(T_{E N D}\right)$ is monotonic increasing with respect to $T_{E N D}$.

Remark 4. It is now possible to explicitly calculate, for a given $\rho_{0}$, the time interval $T_{N}$ such that $\tilde{I}\left(T_{N}\right)=1-\rho_{0}$. Assuming that (9) is solved for a sufficiently large $T_{E N D}$, the following must hold:

$$
\rho_{0} \cdot \tilde{\rho}_{I}\left(T_{E N D}-T_{N}\right)=1-\rho_{0}
$$

We can now state this result for the original optimization problem:

Theorem 2. The optimal $T^{*}(t)$ for the optimization problem (8) can be computed according to the following formula:

$$
T^{*}(t)=\underset{T \in\left(0, T_{N}-t\right]}{\arg \min }\left[\int_{0^{+}}^{T_{N}-t} \eta(s, T)\left[1+\tilde{\rho}_{I}(t+s)\right] d s\right]
$$

Proof. If $T^{*}(t)$ is not the solution to the optimization problem, there exists $\rho_{M A X}$ and $T_{M A X}>T_{N}$ such that $P(t)=P_{\text {des }} \forall t \in\left[0, T_{M A X}\right]$ and:

$$
1-\rho_{0}=\int_{0^{+}}^{T_{M A X}} \rho_{M A X}(t) d t
$$

but this is clearly impossible since the following must also hold:

$$
\int_{0^{+}}^{T_{M A X}} \rho_{M A X}(t) d t \geq \tilde{I}\left(T_{M A X}\right)>\tilde{I}\left(T_{N}\right)=1-\rho_{0}
$$

\section{SIMULATION RESULTS}

The performance of the scheduling described in section 3 has been evaluated through simulations. Initially the turbines dynamics and equation (20) have been discretized. The function $\eta(t, T)$ has been calculated, using two different time vectors and time steps. For the first argument the interval $\left[0, T_{E N D}\right]$ has been chosen, with a time step $\Delta t_{1}=0.01 \mathrm{~s}$, in order to properly capture the dynamics of the switching turbines. For the second argument, which represents the times at which switching back is considered, a bigger time step $\Delta t_{2}=0.5 \mathrm{~s}$ has been used, in order to reduce the computational burden. For the same reason a shorter time vector has been chosen, excluding the time instants that were too close to $T_{E N D}$ and that, after direct verification, represented suboptimal switching times for the turbines. The solution $(\tilde{T}, \tilde{\rho})$ of problem (9) for $T_{E N D}=500 \mathrm{~s}$ has been calculated using the parameters in Slootweg et al. (2001):

$$
\begin{array}{cc}
R=37.5 \mathrm{~m} & J=5.9 \cdot 10^{6} \mathrm{Kg} \cdot \mathrm{m}^{2} \\
v=10 \mathrm{~m} / \mathrm{s} & \omega_{0}=1.69 \mathrm{rad} / \mathrm{s} \\
T_{e q}=K_{T} \omega_{0}^{2}=7.02 \cdot 10^{5} \mathrm{Nm} P_{e q}=K_{T} \omega_{0}^{3}=1.18 \cdot 10^{6} \mathrm{~W}
\end{array}
$$

Notice that $\tilde{\rho}_{I}(t)$ can be evaluated for decreasing values of time, starting with $t=T_{E N D}$, and $\tilde{T}(t)$ is the result, at each time step, of the minimization problem (19). The resulting $\tilde{\rho}(t)$ and its integral, with $\rho_{0}=4.3 \cdot 10^{-4}$, are shown in Fig. 1 and Fig. 2 where they are compared with the case of $T(t)=T_{E N D}$. The optimal values $\tilde{T}(t)$ for 
different torque steps $\tau$ (considered a fraction of the torque $T_{e q}$ in normal operative conditions) are shown in Fig. 3 while the values of $T_{N}$ with respect to $\Delta P / P_{0}$ and the applied torque step $\tau$ are in Fig. 4.

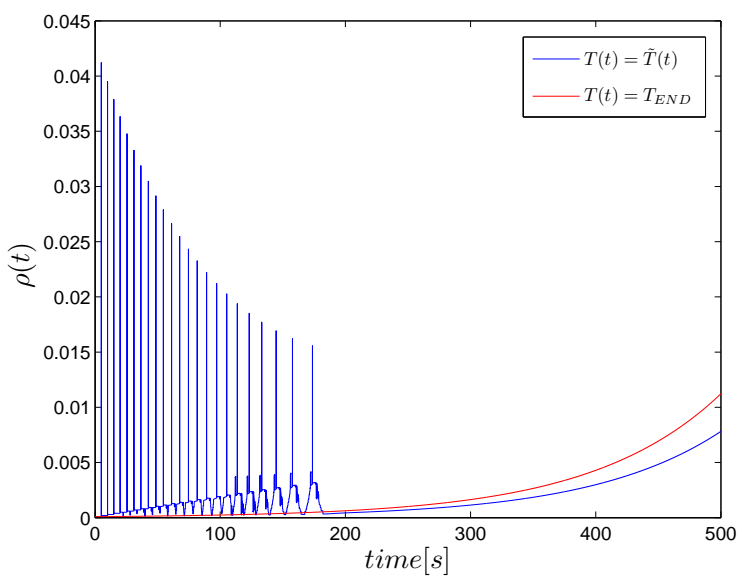

Fig. 1. Values of $\tilde{\rho}(t)$ and $\rho(t)$ with $T(t)=T_{E N D}$. The spikes in the first case are due to the cascaded compensations of the initial fraction of turbines $\rho_{0}$ switching back to mode 1 .

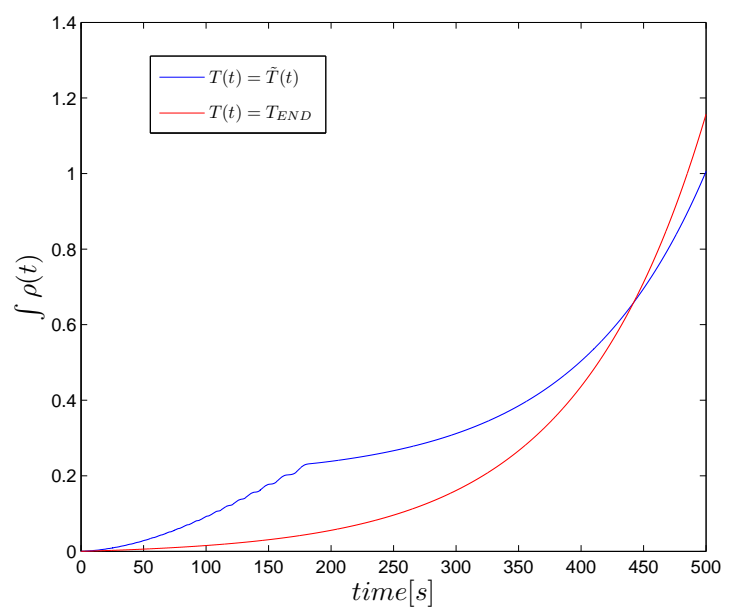

Fig. 2. Integral of $\tilde{\rho}(t)$ and $\rho(t)$ with $T(t)=T_{E N D}$. The integral in the first case is initially bigger, since some of the turbines are switching back and require higher power compensation. When $T_{E N D}$ is approaching the turbines stop switching and, since some of them are again in the first mode of operation (with higher efficiency) $\tilde{\rho}(t)$ increases more slowly.

\section{SCHEDULING WITH UNCONSTRAINED TORQUE}

In the previous sections all turbines can operate in two modes, to which correspond two different expressions for the applied electric torque $T_{e}$. The possibility to arbitrarily set $T_{e}$ for each turbine is now considered. Furthermore, we also suppose that the rotor speed $\omega$ can always be measured, allowing to generate the desired power $P_{e}$ (the new control variable) by setting $T_{e}=P_{e} / \omega$. The study is

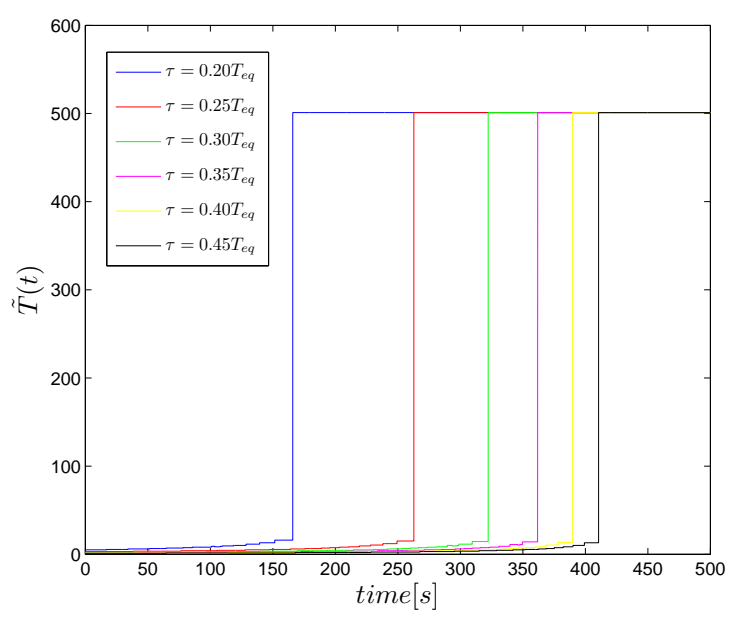

Fig. 3. Values of $\tilde{T}(t)$ for different torque steps, assuming $T_{E N D}=500 \mathrm{~s}$. In all cases, $\tilde{T}(t)$ increases with time: for lower times turbines switch back to the first mode since the instantaneous increase of $\tilde{\rho}(t)$ is compensated over time by the increasing efficiency. There exists a threshold after which is not possible to perform such compensation and turbines remain in the second state $\left(\tilde{T}(t)=T_{E N D}\right)$.

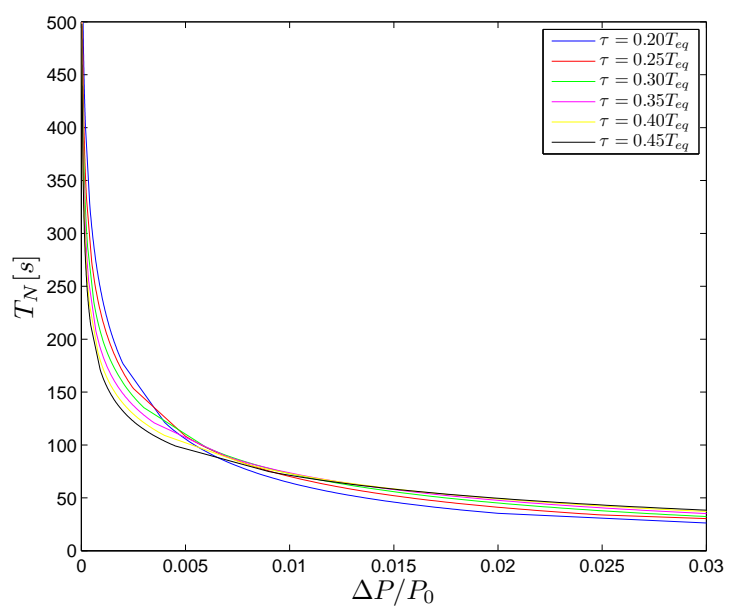

Fig. 4. Values of $T_{N}$ for different power variations and torque steps. Considering that $\rho_{0}=\frac{\Delta P}{\tau \omega_{0}}$, it appears that higher values of $T_{N}$ are achieved in a certain range of $\rho_{0}$ : when $\Delta P$ is lower, low values of $\tau$ are preferable and vice versa.

carried out on a finite number of turbines, which initially operate in steady-state at the optimal rotor speed $\omega_{0}$, generating the total power $P_{0}$. Following a fault in the network, the wind farm is asked to generate the total power $P_{T O T}=P_{0}+\Delta P$. A change of coordinates is introduced in the model of the turbine (1), considering the kinetic energy $E$ as the new state of the system:

$$
\dot{E}=J \omega \dot{\omega}=P_{m}-P_{e}=\tilde{C}_{P}(E)-P_{e}
$$

In (36), assuming constant wind speed, the mechanical power $P_{m}$ is exclusively dependent from the kinetic energy $E$ and will be denoted by $\tilde{C}_{P}(E)=P_{w} C_{P}\left(\sqrt{\frac{2 E}{J}}\right)$ while 
$P_{e}$ represents the generated electrical power. A possible approach to the problem is to maximize the time during which the constant power $P_{T O T}$ is generated and the energy is within the operational limits $\left[E_{M I N}, E_{0}\right]$. Notice that, since turbines are slowing down and releasing part of their kinetic energy, this corresponds to a final state constraint $E_{i}\left(t_{E N D}\right)=E_{M I N}$. If we denote by $P_{i}$ the power generated by each turbine, we can then introduce the following optimal control problem:

$$
\begin{array}{lll} 
& \min _{P_{i}(\cdot), i=1 \ldots N} \int_{0}^{t_{E N D}}-1 d t & \\
\text { s.t. } & E_{i}(0)=E_{0} & \forall \mathrm{i}=1 . . N \\
& E_{i}\left(t_{E N D}\right)=E_{M I N} & \forall \mathrm{i}=1 . . N \\
\dot{E}_{i}(t)=\tilde{C}_{P}\left(E_{i}(t)\right)-P_{i}(t) & \forall \mathrm{i}=1 . . N \\
& \\
\sum_{i=1}^{N} P_{i}(t)=P_{T O T} &
\end{array}
$$

Some indications about the solution of (37) can be obtained by the following static optimization problem, which returns the distribution of energy among the turbines that minimizes the energy losses (i.e. maximize the derivative of total energy):

$$
\begin{aligned}
\underset{\max _{i}, i=1 \ldots N}{\max } & \sum_{i=1}^{N} \tilde{C}_{P}\left(E_{i}\right)-P_{T O T} \\
\text { s.t. } & \sum_{i=1}^{N} E_{i}=E_{T O T} \\
& E_{i} \geq E_{M I N}
\end{aligned}
$$

By introducing this optimization problem, it is now possible to state the following:

Theorem 3. We consider $E_{i}^{*}(t), i=[1 \ldots N]$ as piecewise continuous functions with a finite number of discontinuities such that:

$$
\sum_{i=1}^{N} \dot{E}_{i}(t)=\sum_{i=1}^{N} \tilde{C}_{P}\left(E_{i}(t)\right)-P_{T O T}
$$

If such functions at each time $t$ are a solution to (38) with $E_{T O T}=\sum_{i=1}^{N} E_{i}^{*}(t)$, they are optimal trajectories for the problem (37).

Proof. Notice that two state-vectors $E^{a}=\left[E_{1}^{a}, \ldots, E_{N}^{a}\right]$ and $E^{b}=\left[E_{1}^{b}, \ldots, E_{N}^{b}\right]$ taken as initial conditions have the same optimal cost for (37) if:

$$
\sum_{i=1}^{N} E_{i}^{a}=\sum_{i=1}^{N} E_{i}^{b}
$$

It is in fact possible to instantly switch from one state to another, considering that all $P_{i}$ are unconstrained and the total power required for the switching is equal to zero. It is straightforward to show that, given $E^{*}(t)$ defined in the theorem claim as a solution to $(38)$ and an arbitrary feasible solution $E^{a}(t)$ with $E^{*}(0)=E^{a}(0)$, the following holds:

$$
\sum_{i=1}^{N} E_{i}^{*}(t) \geq \sum_{i=1}^{N} E_{i}^{a}(t) \forall t>0
$$

Notice that $E_{i}^{*}\left(t_{E N D}\right)=E_{M I N} \forall i$, therefore we have:

$$
\sum_{i=1}^{N} E_{i}^{*}\left(t_{E N D}\right)=N E_{M I N} \geq \sum_{i=1}^{N} E_{i}^{a}\left(t_{E N D}\right)
$$

All components of $E^{a}\left(t_{E N D}\right)$ are equal to $E_{M I N}$ (or there would exist one which is lesser than $\left.E_{M I N}\right)$, therefore $E^{a}$ is never better than $E^{*}$ for the optimal problem (37).

We now turn our attention to the resolution of (38) for a given value of total energy $E_{T O T}$. Consistently with our original choice of $C_{P}$, we assume that $\frac{\partial \tilde{C}_{P}(E)}{\partial E}$ is always positive in $\left[E_{M I N}, E_{0}\right]$ and its only stationary point is a maximum for $E=E_{T H R}$. In other words, the second partial derivative of $\tilde{C}_{P}(E)$ changes sign for $E=E_{T H R}$. It is now possible to state the following result:

Theorem 4. Under the current assumptions on $\tilde{C}_{P}(E)$, the vector $\bar{E}=\left[\frac{E_{T O T}}{N}, \ldots \frac{E_{T O T}}{N}\right]$ is a local maximum of $(38)$ if $E_{T O T}>N \cdot \stackrel{N}{E}_{T H R}$.

Proof. The Lagrangian of the optimization problem, denoted by $L(E, \lambda)$, has the following expression:

$$
L(E, \lambda)=\sum_{i=1}^{N} \tilde{C}_{P}\left(E_{i}\right)+\lambda\left(E_{T O T}-\sum_{i=1}^{N} E_{i}\right)
$$

The first order condition for the existence of a local optimum reads as follows:

$$
\frac{\partial L(E, \lambda)}{\partial E_{i}}=\frac{\partial \tilde{C}_{P}\left(E_{i}\right)}{E_{i}}-\lambda=0
$$

Notice that, according to (43), $\frac{\partial \tilde{C}_{P}\left(E_{i}\right)}{\partial E_{i}}$ must be the same for all $i$ : such condition is verified for $E_{i}=\frac{E_{T O T}}{N}$. We now consider the second order necessary condition (SOC) which states, in this case, that the stationary point is a local maximum if, starting with the principal minor of order 3, the last $N-1$ principal minor determinants of the bordered Hessian alternate in sign, starting with $(-1)^{2}$. For the considered problem the bordered Hessian is:

$$
H=\left[\begin{array}{cccc}
0 & -1 & \cdots & -1 \\
-1 & \frac{\partial^{2} \tilde{C}_{P}\left(E_{1}\right)}{\partial E_{1}^{2}} & & \\
\vdots & & \ddots & \\
-1 & & & \frac{\partial^{2} \tilde{C}_{P}\left(E_{N}\right)}{\partial E_{N}^{2}}
\end{array}\right]
$$

and the determinant of the principal minor of order $n+2$, evaluated at $E_{i}=\frac{E_{T O T}}{N}$, has the following expression:

$$
-(n+1)\left[\frac{\partial^{2} \tilde{C}_{P}\left(\frac{E_{T O T}}{N}\right)}{\partial E^{2}}\right]^{n}
$$

The proof is concluded by noticing that, under the current assumption on $E_{T O T}, \frac{\partial^{2} \tilde{C}_{P}\left(\frac{E_{T O T}}{\partial E^{2}}\right)}{\partial s}$ negative and the $\mathrm{SOC}$ is satisfied.

The results presented above have been verified through simulations. Using the parameters in (35) and considering a number of turbines $N=10$, the numerical solution to the maximization problem has been calculated. In this case the energy coefficient $\tilde{C}_{P}(E)$ satisfies the necessary conditions of the local maximum for all $E>E_{T H R}$, with $E_{T H R}=1.47 \cdot 10^{6} \mathrm{~J}$. In Fig. 5 the global optima $E_{i}^{*}$ are shown for different values of $E_{T O T}$. It is possible to notice from the figure that, for high values of total energy, local and global maximum coincide. On the other hand, there exists a threshold energy $E_{G}$ (dotted blue line) greater 


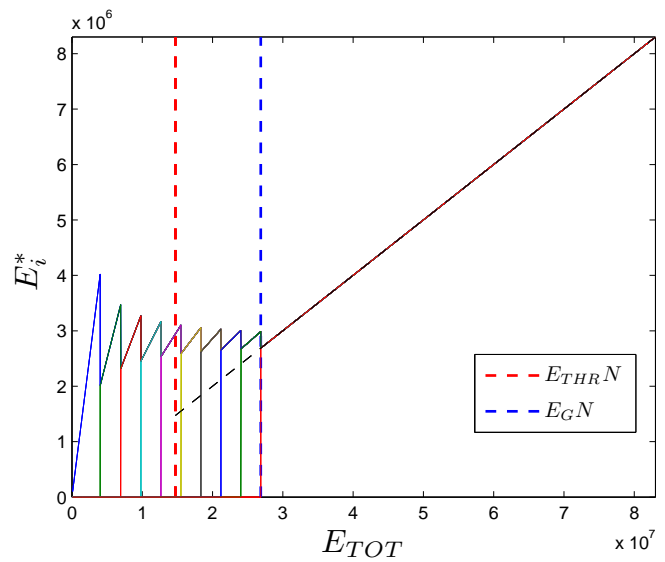

Fig. 5. The optimal values $E_{i}^{*}$ as functions of $E_{T O T}$. The continuous coloured lines show the global maximum for the different turbines while the black dotted line represents the local maximum $E_{i}=E_{T O T} / N$ expected from the second order conditions.

than $E_{T H R}$ (dotted red line) for which a new global maximum appears: it is more convenient to instantly slow down one turbine and equally redistribute its energy content among the remaining turbines. It should be pointed out that, for the value of $E_{G}$ obtained with the considered parameters, the corresponding speed seems to be smaller than the minimum rotor speed at which wind turbines are usually operated. Therefore, an uniform distribution of the energy among the turbines appears to be the optimal solution in most of the cases. In this situation the optimal policy can be implemented in a decentralized way: each turbine will be able to independently detect the fault and provide a proportional extra power, with the proportional constant equal for all turbines.

We are now interested in comparing the optimal policy for unconstrained torque with the one of Section 3, where the turbines can only switch between two operative modes. The comparison is made by considering the optimal time in both cases, when the same variation of total kinetic energy is introduced and the same amount of extra power is produced. The kinetic energy $E_{k}$ for the constrained case after $T_{N}$ seconds has been calculated with the same parameters used in the simulations of Section 4. Denoting with $\omega(t, T)$ the rotor speed at time $t$ of a turbine that switches back to the original mode after $\mathrm{T}$ seconds we have:

$$
E_{k}=\frac{1}{2} J \int_{0}^{T_{N}} \rho^{*}(t) \cdot \omega^{2}\left(T_{N}-t, T^{*}(t)\right) d t
$$

The optimal time $\bar{T}_{N}$ in the unconstrained case has been calculated by producing the same quantity of extra power and optimally slowing down the turbines until the same value $E_{k}$ of kinetic energy is achieved. The ratio $r_{T}=\frac{T_{N}}{T_{N}}$ is shown in Fig. 6.

\section{CONCLUSIONS}

In this paper a scheduling of wind turbines for frequency control is presented. In the initial section the single turbine is modelled and the expression for the power extracted from the wind is calculated. The scheduling problem is initially solved when turbines can operate in two different

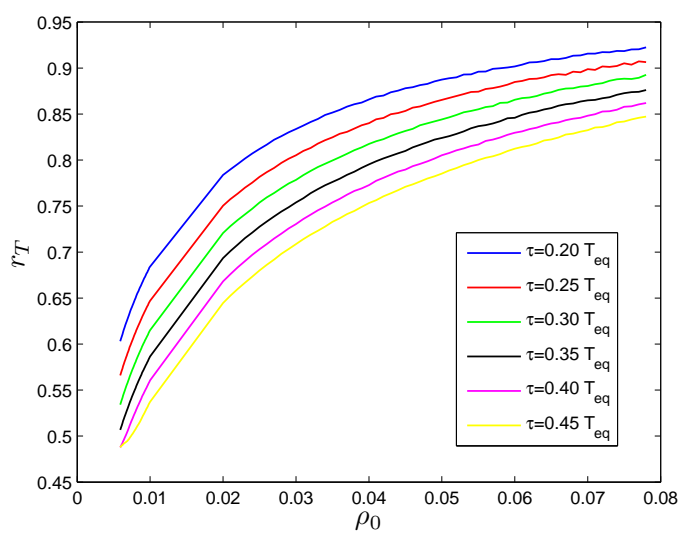

Fig. 6. Ratio $r_{T}$ of the optimal times in the two cases, for different values of the parameters $\tau$ and $\rho_{0}$. For high values of $\rho_{0}$, the repartition of power among the turbines in the two-modes scenario closely resembles the one of the unconstrained case, therefore their performances are more similar.

modes, providing an optimal policy which maximizes the frequency support time from wind generation and can be easily calculated numerically. The problem with unconstrained electric torque has also been considered, showing that in this case optimality is obtained by equally distributing the torque among the turbines until a threshold rotor speed is reached.

\section{REFERENCES}

Boué, M. and Dupuis, P. (2001). Risk-sensitive and robust escape control for degenerate diffusion processes. Mathematics of Control, Signals and Systems, 14, 62-85.

Burton, T., Jenkins, N., Sharpe, D., and Bossanyi, E. (2001). Wind energy handbook. John Wiley \& Sons, Chichester, UK.

de Almeyda, R. and Lopes, J.P. (2007). Participation of doubly fed induction wind generators in system frequency regulation. IEEE Transactions on Power Systems, 22(3), 944-950.

Falugi, P., Kountouriotis, P., and Vinter, R.B. (2012). Differential games controllers that confine a system to a safe region in the state space, with applications to surge tank control. IEEE Transactions on Automatic Control, 57(11), 2778-2788.

Kundur, P. (1994). Power stability and control. McGraw Hill, New York.

Lalor, G., Mullane, A., and O'Malley, M. (2005). Frequency control and wind turbine technologies. IEEE Transactions on Power Systems, 20(4), 1905-1913.

Ogawa, S., Allison, B., Dumont, G., and Davies, M. (2002). A new approach to optimal averaging level control with state constraints. Proceedings of 41 st Conference on Decision and Control, 2, 1952-1957.

Slootweg, J., Polinder, H., and Kling, W.L. (2001). Dynamic modelling of a wind turbine with doubly fed induction generator. Power Engineering Society Summer Meeting, 2001, 1, 644-649.

Ullah, N., Thiringer, T., and Karlsson, D. (2008). Temporary primary frequency control support by variable speed wind turbines - potential and applications. IEEE Transactions on Power Systems, 23(2), 601-612. 